\title{
A NEW HIGH-RESOLUTION OPTICAL STUDY OF HALO GAS
}

C. E. Albert

U.S. Naval Academy, Annapolis, MD, USA

J. C. Blades

Space Telescope Science Institute, Baltimore, MD, USA

D. C. Morton and M. Proulx

Herzberg Institute of Astrophysics, Ottawa, ON, Canada

F. J. Lockman

National Radio Astronomy Observatory, Charlottesville, VA, USA

\begin{abstract}
We present preliminary results from a new high resolution optical study of halo gas at the coudé focus of the Canada - France - Hawaii Telescope. Our work is still in progress so two general results are presented here: significant absorption is produced in interstellar gas beyond $500 \mathrm{pc}$ from the galactic plane, and well-resolved halo clouds are identified.
\end{abstract}

1. Introduction.

The identification and study of interstellar clouds in the galactic halo has been a subject of interest since the pioneering work of Münch and Zirin in 1961. They measured the velocities and intensities of absorption lines of Ca II and, in some cases, $\mathrm{Na}$ I toward 24 high latitude $\mathrm{OB}$ stars ranging from $200 \mathrm{pc}$ to $2 \mathrm{kpc}$ beyond the plane. Their observations showed a real increase with distance in the number of $\mathrm{Ca}$ II components per star, implying that some of the observed gas must be located at distances up to a kiloparsec from the galactic plane. This important study prompted further work by, among others, Greenstein (1968), Rickard (1972), Richstone and Morton (1975) and Cohen and Meloy (1975). 
pc, HD 123884 shows two strong Ca II components, implying that substantial amounts of gas must be located between $420-1000 \mathrm{pc}$. The same structure is seen toward HD 119608 and is echoed in the strong Ti II line. All lines show additional weak unresolved components. However, the equivalent width of Ca II toward HD 123884 is stronger than that toward the more distant HD 119608 so the interstellar gas is evidently quite clumpy and relatively little material seems to exist beyond $1000 \mathrm{pc}$. These lines of sight are plotted in Figure 2: we seem to be detecting a large inhomogeneous cloud well below the star at $z=2600 \mathrm{pc}$, but above $420 \mathrm{pc}$. This example illustrates two major results of our study: an increase in integrated interstellar equivalent width and total absorption velocity range beyond the galactic plane and the identification of a set of discrete clouds with good distance limits. The latter will be a major focus of our abundance analysis.

FIG. 1. - Interstellar absorption lines along the line of sight toward HD 119608 and foreground stars. All continua are normalized to unity and the length of the vertical scale from 0 to 1 is the same for all spectra.

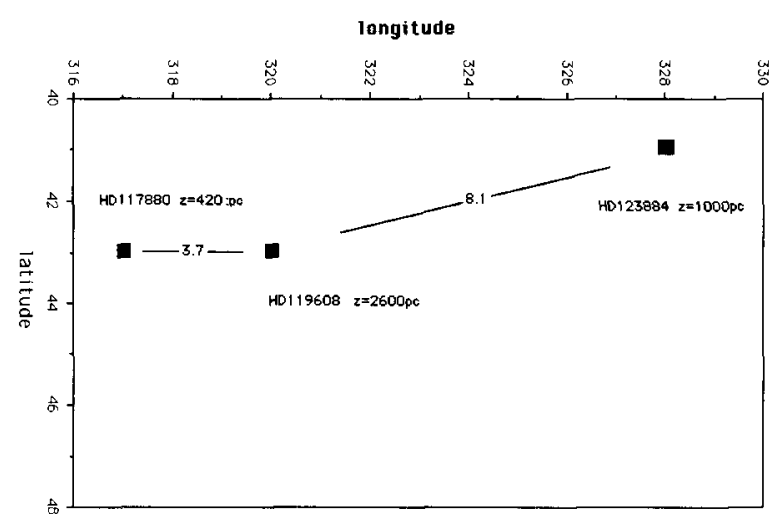

HDI 19608 Field

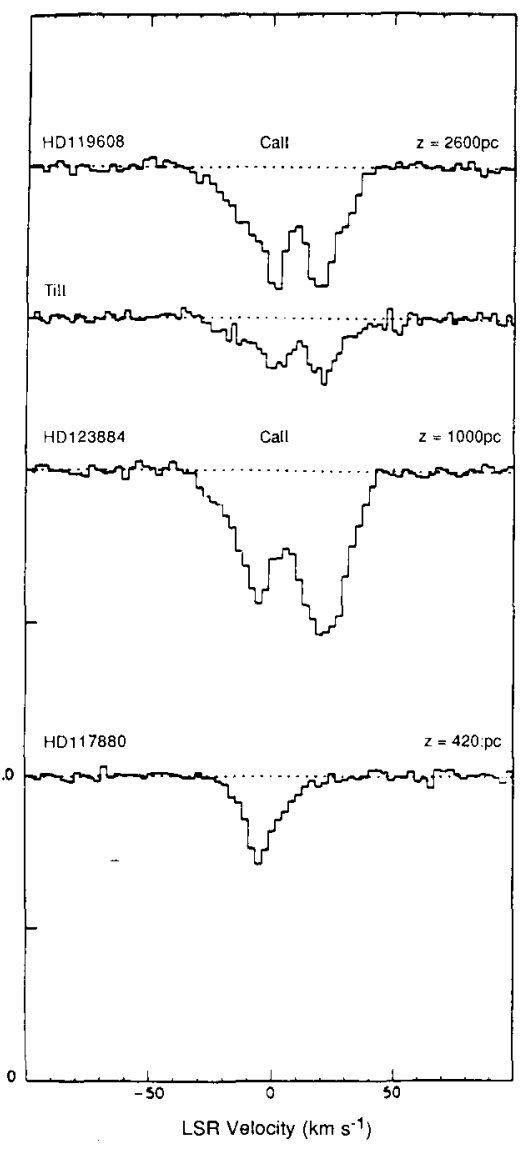

FIG. 2. - HD 119608 field. The separation between stars is shown in degrees. 
There is a clear increase in both the total equivalent widths and the absorption velocity ranges from the average distance to the foreground stars $(\mathrm{z} \approx 145 \mathrm{pc})$ to the average distance to the halo stars $(\mathrm{z}=1100 \mathrm{pc})$. Prominent low-velocity absorption is observed in all directions, but higher velocity gas appears beyond the foreground stars, including several discrete high velocity clouds at both positive and negative velocities. Figure 3 depicts observations of the $\mathrm{K}$ line of $\mathrm{Ca}$ II toward all the stars, where the data are averaged in three distance ranges: $\mathrm{z}<500 \mathrm{pc}, 500<\mathrm{z}<1000 \mathrm{pc}$ and $\mathrm{z}>1000 \mathrm{pc}$. There is substantial material between $500-1000 \mathrm{pc}$, but not significant gas beyond a kiloparsec. This confirms our earlier work.

FIG. 3. - Integrated equivalent widths of the $\mathrm{K}$ line of $\mathrm{Ca}$ II toward 64 high latitude stars, averaged for 3 distance ranges: $z<$ $500 \mathrm{pc}, 500<\mathrm{z}<1000 \mathrm{pc}$ and $\mathrm{z}>1000 \mathrm{pc}$. The vertical height of each bar is proportional to the total observed equivalent width within the indicated velocity ranges. The small symbol in the upper left corner denotes the height corresponding to an equivalent width of $100 \mathrm{~mA}$.

\section{Discrete Halo Clouds.}

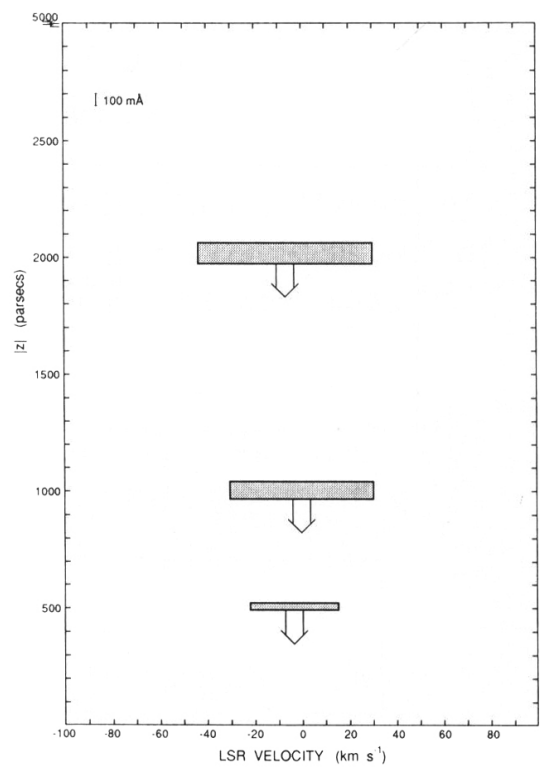

Twenty-three good cases of individual high latitude Ca II clouds have been identified, including several examples of clouds well outside the galactic plane. These are resolved clouds, chosen outside the average velocity range of absorption toward stars with $\mathrm{z}<500 \mathrm{pc}\left(-22 \leq v_{\mathrm{LSR}} \leq+14 \mathrm{~km} / \mathrm{s}\right)$. In 10 cases, the velocity exceeds \pm 70 $\mathrm{km} / \mathrm{s}$ and six of these are in directions showing high velocity $\mathrm{H}$ I clouds in Wakker's (1989) survey (also Hulsbosch and Wakker 1988). The signs of the Ca II cloud velocities are the same as those observed in $\mathrm{H} \mathrm{I}$.

The Ca II absorption lines are generally weak, with $\log \mathrm{N}(\mathrm{Ca}$ II) typically ranging from $10-11$, but a few strong components are observed. Some of these $\mathrm{Ca}$ II clouds are detected in the $21 \mathrm{~cm}$ spectra while others are not. It is likely that all of these clouds have low H I column densities. For example, for cosmic abundances, even if all 


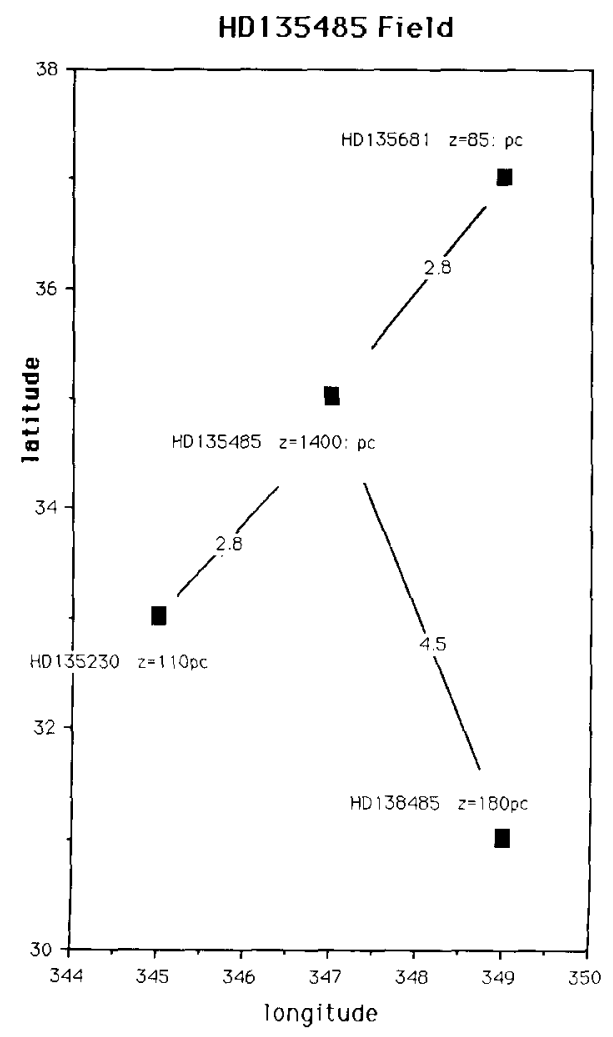

FIG. 4. - HD 135485 field. The separation between stars is shown in degrees.

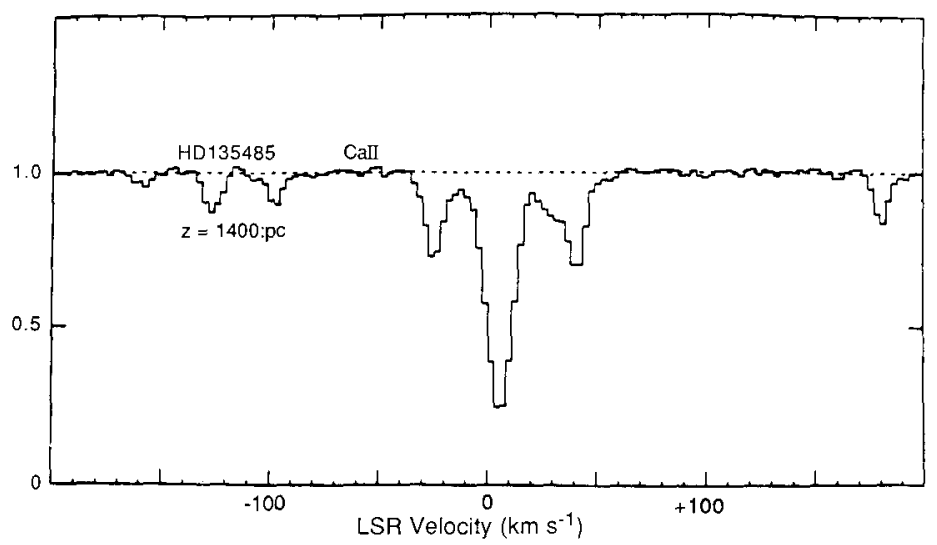

FIG. 5. - The interstellar Ca П $\mathrm{K}$ line along the line of sight toward HD 135485.

the calcium in the cloud toward HD $3379(\log \mathrm{N}(\mathrm{Ca}$ II $)=10.54)$ were singly ionized, the corresponding $\mathrm{H}$ I column density of $\log \mathrm{N}(\mathrm{H} \mathrm{I}) \approx 17$ would be too low for us to detect. Thus, these observations alone do not allow us to conclude whether the clouds are neutral or ionized. 
A basic conclusion of this work is that we do not find a significant population of massive high velocity clouds: there are certainly several interesting individual cases, but they seem to contain little mass. This material also appears to be quite patchy with a great deal of spatial structure.

The line of sight toward HD 135485, originally studied by Münch and Zirin, provides a good example: the observed field is plotted in Figure 4 and the $\mathrm{Ca} I \mathrm{~K}$ spectrum toward HD 135485 is shown in Figure 5. Only weak low velocity $\mathrm{Ca}$ II is detected toward the three foreground objects in this direction. However HD 135485, 1400: pc above the plane, shows complex and strong $\mathrm{Ca}$ II over a wide velocity range. At low velocities, the $21 \mathrm{~cm} \mathrm{H} \mathrm{I} \mathrm{emission} \mathrm{is} \mathrm{consistent} \mathrm{toward} \mathrm{all} \mathrm{four} \mathrm{stars,} \mathrm{implying}$ that a large cloud covers much of this area. In HD 135485 there are spectral lines which, if attributed to interstellar Ca II K, would have LSR velocities of -98 and -128 $\mathrm{km} / \mathrm{s}$. These are not detected toward the foreground stars in this field. The features have possible, but very weak, $\mathrm{H}$ I emission counterparts. For depleted disk clouds the observed $\log \mathrm{N}(\mathrm{Ca}$ II $)=11$ would be easily detected at $\mathrm{H}$. These halo clouds appear to be significantly less depleted than typical disk clouds, which is consistent both with our earlier work and with Danly's (1989) ultraviolet observations toward halo stars.

\section{REFERENCES}

Albert, C. E. 1983, Ap.J., 272, 509.

Cohen, J. G., and Meloy, D.A. 1975, Ap.J., 198, 545.

Danly, L. 1989, this volume.

Greenstein, J. L. 1968, Ap.J., 152, 431.

Hulsbosch, A. N. M., and Wakker, B. P. 1988, Astr. Ap. Suppl. Ser., 75, 191.

Morton, D. C., and Blades, J. C. 1986, Mon. Not. R. astr. Soc., 220, 927.

Münch, G., and Zirin, H. 1961, Ap.J., 133, 11.

Richstone, D. O., and Morton, D. C. 1975, Ap. J., 201, 289.

Rickard, J. J. 1972, Astr. Ap., 17, 425.

Wakker, B. 1989, this volume.

Wallerstein, G., and Goldsmith, D. 1974, Ap.J., 187, 237.

\section{Discussion:}

MÜNCH (Comment): Your IS line profiles in HD119608 seem to show distinct extended wings, not apparent in the lower resolution and S/N-ratio photographic observations I made 30 years ago. Their appearance suggests their origin is a superposition of many weak unresolved lines. From the behaviour of the $\mathrm{Ca}$ II and $\mathrm{Na}$ I lines arising in high velocity clouds, Spitzer and Routly, first, and later myself, suggested a relation between the kinematics of clouds and their physical state. On basis of your observations, we should be able to establish in a more quantitative fashion that relation between kinematics (or distance to the galactic plane) and degree of ionization, electron temperature, etc. 